\title{
Saccadic localization of visual targets by the very young human infant
}

\author{
RICHARD N. ASLIN and PHILIP SALAPATEK \\ University of Minnesota, Minneapolis, Minnesota 55455
}

\begin{abstract}
The direction, latency, and form of the 1- and 2-month-old human infant's saccadic eye movements toward peripheral targets were investigated. Infants of both ages reliably executed a directionally appropriate first saccade toward a peripheral target introduced as far as $30 \mathrm{deg}$ from the line of sight along the horizontal and both diagonal axes, but only to $10 \mathrm{deg}$ along the vertical axis. The presence of a second target in the central visual field reduced the probability of peripheral target localization. A significant inverse relation was found between target distance from the line of sight and probability of initiating a directionally appropriate saccade. Electro-oculography revealed that latency to first saccade, although highly variable, was less than $500 \mathrm{msec}$ on a significant proportion of trials. Unlike the adult, the first saccade to target was grossly hypometric and was followed by one or more saccades of approximately equal amplitude to the first.
\end{abstract}

Efficient foveation requires a highly sophisticated spatial localization system capable of rapidly translating the line of sight to predetermined peripheral locations. In the human adult, saccadic eye movements toward a peripheral stimulus are of very high velocity (up to $800 \mathrm{deg} / \mathrm{sec}$, see Alpern, 1971, p. 376), have latencies as brief as 120 to $350 \mathrm{msec}$ dependent upon stimulus conditions (Bartz, 1962; Komoda, Festinger, Phillips, Duckman, \& Young, 1973; Leushina, 1965; Saslow, 1967; Wheeless, Boynton, \& Cohen, 1966; White, Eason \& Bartlett, 1962), are highly accurate (Bartz, 1967; Becker \& Fuchs, 1969; Rashbass, 1961; Weber \& Daroff, 1971), and are ballistic in nature (Westheimer, 1954). The preprogramming of saccadic movements requires the accurate specification of target locus from the current line of sight and the translation of this information into motor commands resulting in a direction- and distance-appropriate eye movement.

In spite of the vast amount of research on the oculomotor system in animals and human adults (e.g.. Bach-Y-Rita \& Collins, 1971; Davson, 1972; Dichgans \& Bizzi, 1972; Grunin \& Mostofsky, 1968), few attempts have been made to investigate in detail the saccadic system of the human infant. Although it appears that the young human infant executes saccades during alert viewing of stationary figures (Kesson, Salapatek, \& Haith, 1972; Salapatek, 1968;

This research was partially supported by Grant HD-05027 to the Institute of Child Development and by Grants HD-01136 and NSF P2B1389 to the Center for Research in Human Learning. We gratefully acknowledge the assistance of Emily Bushnell. Elliot Saltzman, and John Simonson, who acted as observers, and to Mary Jayne Capps, Marshall Haith, Herschel Leibowitz, Davida Teller, and James Zacks, who provided a critical reading of an earlier draft. Requests for reprints should be addressed to Richard N. Aslin, Institute of Child Development, University of Minnesota, Minneapolis, Minnesota 55455.
Salapatek \& Kessen, 1966; see Bond, 1972, and Kessen, Haith, \& Salapatek, 1970, for general reviews), an exact description of these movements has not been reported. ${ }^{1}$ In particular, there has been no detailed investigation of the latency, accuracy, and form of infant eye movements toward a single, small peripheral target introduced into an otherwise patternless field.

Tronick and Clanton (1971) investigated eye-head coordination in 4- to 15-week-old infants. However, they provided only a qualitative description of various eye- and head-movement patterns. Their use of multiple targets in the visual array precluded detailed description of (a) directionality of the first eye movement to a peripheral target, (b) amplitudes of localizing saccades, and (c) latency to initiate an eye movement to a peripheral target. Tronick (1972) investigated the time taken by 2 - to 10 -week-old infants to fixate a single peripherally introduced target, but did not report the form of the eye movements executed during localization. Moreover, a fixated central stimulus remained on after the introduction of the peripheral target. He found that infants took an average of approximately 3 to $5 \mathrm{sec}$ to localize the target. This long average latency may have resulted from the attentional "capture" of the infant's gaze by the central fixation stimulus, which has been reported by several infant investigators (Ames \& Silfen, Note 1; Mundy-Castle \& Anglin, Note 2; Salapatek, Note 3; Stechler \& Latz, 1966). In a more recent study, Harris and MacFarland (1974) showed that even newborn infants would shift their gaze towards the side of the visual field in which a peripheral target was introduced. Moreover, targets were localized at a greater distance if a central stimulus which the subjects were initially fixating disappeared at the time of peripheral target onset 
than if the central stimulus remained on. Although this study strongly suggests some directional speciticity in the saccadic system at birth. the nature of their recording of localizing saccades (human observers) precludes any statements regarding the latency, amplitude, or form of a localizing saccade.

At birth, the localization of a peripheral target may be accomplished as in the adult. That is, retinal local signs for target direction and angular distance may result in highly accurate and ballistic first saccades. Alternatively, there may be neither local signs nor effective motor mechanisms to insure direction- and distance-appropriate localizing saccades. Although the Harris and MacFarlane study indicates some directional specification at birth, the development of accurate saccadic localization might proceed by a learning and/or maturation process involving either the receptive or the motoric systems, or both. For example. McLaughlin (1967) has shown that adults are capable of rapidly recalibrating the distance component of the saccadic system, but there are no human infant data indicating either that initial appropriate distance calibration exists or that rapid recalibration of this component is possible. From anatomical evidence, it would appear that the saccadic system must undergo some developmental calibration due to (a) shifts in retinal cell loci relative to the optic axis (Mann, 1964), (b) large increases in eyeball mass (Todd, Beecher, Williams, \& Todd, 1940), and (c) increases in eyeball diameter which may be accompanied by differential alteration in the dimensions of the various refractive surfaces of the eye (Wilmer \& Scammon, 1950). These changes make it very unlikely that either retinal local signs or a fixed motor program specifying target distance and direction remain invariant during development.

This study describes the probability, latency, direction, and form of the infant's eye movements toward a peripheral target replacing or added to a central fixation stimulus. To assess these parameters accurately, electro-oculography (EOG) as well as direct observation, was employed.

\section{METHOD}

\footnotetext{
Subjects

Twenty-four 1-month-old human infants (12 males and 12 lemales: mean age - 32.5 days, range - 25-37 days) and 24 2-month-olds $(15$ males and 9 females: mean age $=61.0$ days. range - 55-67 days) were sequentially assigned to one of four experimental groups. All subjects were Caucasian, home reared, ahove $2.750 \mathrm{~g}$ at birth, and obtained on a volunteer basis by phone solicitation based on newspaper birth announcements. An additional to subjects were eliminated from the study as follows: 11 1 -month-olds (1 slecepy. 9 inattentive to a central tixation stimulus, and 1 becaluse of poor observer agreenent) and 5 2-month-olds (1 slecpy and 4 inatlentive to a central fixation stimulus).
}

\section{Apparatus and Stimuli}

Supine ubjects viewed the stimuli by looking at a rear-projection screen reflected into a hall-silvered mirror (see Figure 1). The mirror was tixed at $45 \mathrm{deg}$ with respect to the projection sereen. The viewing distance from the center of the stimulus field to the subject wals approximately $35 \mathrm{~cm}$.

Ihe atpratatus wats designed to display small anmular targets in the ecenter and at vatrous peripheral locations on a dark gray background (average luminance $=2 \mathrm{fL}$ ). Target diameter was $26 \mathrm{~mm}$ (approximately $4 \mathrm{deg}$ at field center), annular width $3 \mathrm{~mm}$. and annular luminance $10 \mathrm{tL}$. One Kodak Carousel projector was used to project a target in the eenter of the stimulus field. A second projector presented targets at any of the 24 kceations peripheral to the center of the stimulus fiedd.

Fath frial consisted of the presentation of at farget in the center of the stinulus tield (central lixation stimulus) followed either by (a) the addlition of a target in the peripheral stimulus tield (addition). or (b) the offset of the central fixation stimulus coincident with the onset of a peripheral target (replacement). Each projector was equipped with an Ilex No. 4 Synchro electronic shutter so that durations of target onset and offset were nearly instantaneous (approximately 8 and $5 \mathrm{msec}$, respectively). In the npherment condition. no pereeptible delay between central lixation stimulus olfiset and peripheral target onset. and no motion or afterimage eflects were experienced by adult observers.

FOG recordings were allempted on all subjects except those receiving stimuli only along the vertical axis of the stimulus field. Pilot work had suggested that electrode placement below the eye for vertical recording was distracting to the infant. Two Beckman miniature biopotential electrodes were attached to the outer camthus of each orbit using Hewlett-Packard Redux electrode paste and mintature adhesive collars. The skin was rubbed with Beck-Lee Microhm jelly and rinsed with distilled water prior to electrode attachment. A third ground electrode was attached to the midforchead in an identical manner. EOG was recorded ac (time constant - $0.6 \mathrm{sec}$ ) on a Gilson Model IC $[-1 \mathrm{H}$ single-channel polygraph. Sensitivity $w a s \mathrm{kept}$ at $500 \mu \mathrm{V} / \mathrm{cm}$ and paper speed at 25 mmisec. enabling EOG potentials to be scored to the nearest $25 \mu \mathrm{V}$ and hatencies to the nearest 40 msee. With this sensitivity. adclut eye movements of 2 deg or more could be detected on the IOOF recosd.

\section{Design}

Addetion and replacement trials were delivered along four axes in Ile simulas tield. Imlependent groups of 12 subjects (o at carch age) were presented with addition and replacement trials along the horizontal, the vertical, or one of the two diagonal axes. Peripheral

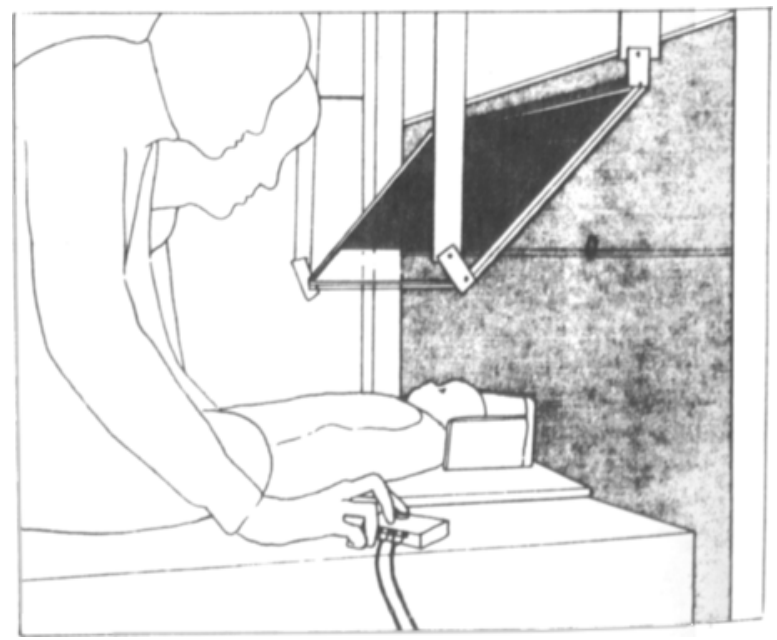

Figure 1. Infant eye movement recording apparatus illustraling the posilioning of the two observers behind the half-silvered mirror. 
targets along the left and right vectors of the horizontal axis were presented al 10. 20. 30, and $40 \mathrm{deg}$ to the left and right. respectively. of the central fixation stimulus: along the vertical rectors at 10 and 20 deg above and below the central fixation stimulus: and along the diagonal vectors at 10.20 , and $30 \mathrm{deg}$ diagonalk from the central fixation stimulus.

All stbiccls al wo received wo types of control trials consisting of either (a) the replacement of the central fixation stimulus by a homogenenus gras field (center-ott). or (b) the addition of a homogenetus gray field to the central fixation stimulus (center-on). In each case. the brightness of the resultant gray background was equal to the brightness of the gray background of a peripheral target trial.

All subjects were required to complete at least two blocks of trials. A block consisted of: one addition trial and one replacement trial at each distance along each vector of a given axis. plus two center-on and two center-otf control trials. Order of trials was random except that each halt-block never contained two trials at the same distance along a given vector.

Two observers were stationed behind the mirror. one on either side of the subject (see Figure 1). A shield on the upper rear portion of the mirror allowed the observers to see the subject's eyes through the lower portion of the half-silvered mirror, but not the targets projected on the screen. The two independent observers simultaneously recorded the direction of gaze of the subject by pressing buttons connected to an event recorder. In addition. one observer attempted to keep the subject's head centered during the experiment. usually with the aid of a honey-coated pacifier. The other observer controlled trial onset.

Each stinulus trial began with the presentation of the central tixation stimulus. When the observer controlling trial onset judged that the subject was staring directiy up at the mirror (and presumably at the central tixation stimulus), an addition. a replacement, or a control trial was introduced. Trial duration was 10 sec. with a 3-sec intertrial interval. followed by the reintroduction of the central fixation stimulus.

The observers knew each subject's axis. the time of trial onset. and that the subject was looking at the central fixation stimulus prior to trial onset. However, the observers were blind as to the direction and distance of the peripheral target from the central fixation stimulus. and the particular condition laddition. r'placement. controf delivered on any given trial. The observers were required only to record. during each 10 -sec trial, whether the subject was siewing the center or one of the two semitields defined by each subject's particular axis. e.g.. whether the subject was looking up. down. or center. if his peripheral targets were delivered along the vertical axis. etc.

\section{RESULTS}

The 1- and 2-month-olds received a total of 916 and 1.030 trials, respectively; more of the 2 -month-olds continued past the two-block minimum. Scorer agreement on the direction of first eye movement was $84 \%$ and did not differ significantly by age, target distance. condition, or axis. All trials on which there was scorer disagreement were eliminated from further analyses.

\section{Direction of First Eye Movement}

A probability ratio for directionally appropriate tirst eve movements was computed for each subject for each peripheral target along his axis. This ratio was the number of trials on which he made a directionally appropriate first eye movement divided by the total number of trials on which that target was delivered. A

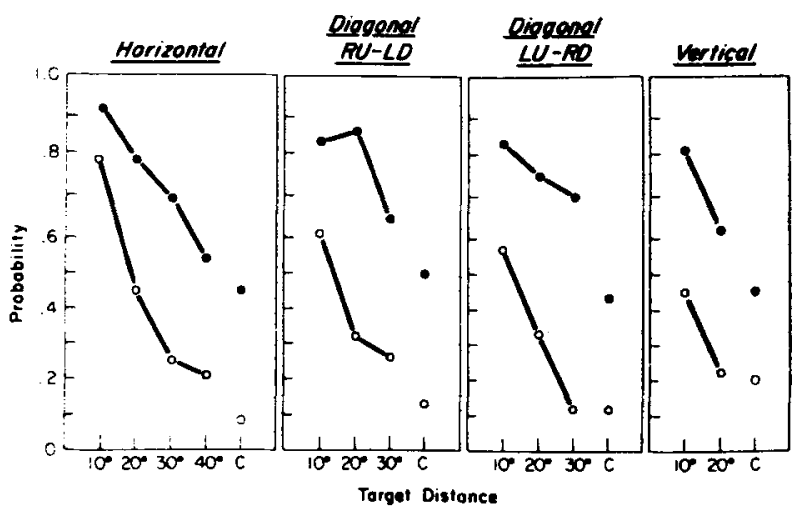

Figure 2. Mean probabilities of making a directionally appropriate first eye movement to peripheral targets, as a function of target distance, for the four axis groups. $\mathbf{C}=$ control trial means. Filled circles = replacement trials; open circles = addition trials, $\mathbf{R U}=$ right-up vector; $\mathbf{L D}=$ kft-down vector; $\mathbf{L U}=$ left-up vector; $\mathbf{R D}=$ right-down vector.

control trial ratio for center-on and for center-off trials was also computed separately for each subject. These ratios consisted of the number of first eye movements along a particular vector divided by the total number of each type of control trials delivered. Thus, for both center-on and center-off trials, there existed for each subject a control trial ratio indicating the proportion of trials on which he executed a first eye movement along a particular vector by chance.

Figure 2 presents the mean probabilities of a directionally appropriate first eye movement as a function of peripheral target distance from the central fixation stimulus for addition, replacement, and control trials. Although replacement trial means were consistently higher than addition trial means across all axes and distances, it should be noted that center-off and center-on means (control baselines) appear to differ by a similar amount. ${ }^{2}$ A strong distance effect was also indicated for both addition and replacement trials.

To test these trends, four analyses of variance, one on each axis group of 12 subjects, were computed. Appropriate control trial ratios were subtracted from experimental trial ratios for each subject to eliminate the probability of chance responding. No consistent effects of age, vector, or addition-replacement ${ }^{3}$ were found. In all four analyses, however, there was a significant main effect for distance (horizontal, $\mathrm{p}<.0005$; diagonal RU-LD, $\mathrm{p}<.005$; diagonal LU.RD, $p<.001$; vertical, $p<.01$ ). As Figure 2 indicates. the subjects responded more reliably to near than to far targets. Dunnett's $t$ statistic $(a<.025)$ indicated higher experimental than control eye-movement probability at 10,20 , and $30 \mathrm{deg}$ along the horizontal and both diagonal axes, but only at $10 \mathrm{deg}$ along the vertical axis. 


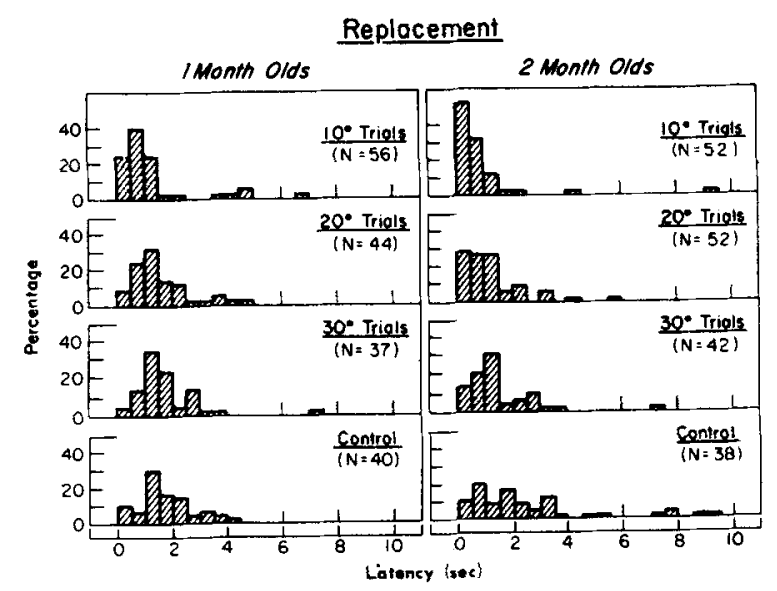

Figure 3. Frequency histogram of EOG latencies, in 500-msec intervals, to initiate a directionally appropriate first eye movement on replaceme'tut trials for 1 - and 2 -month-olds. $\mathbf{N}=$ number of trials.

\section{Latency of First Eye Movement}

EOG recordings were attempted on the 36 horizontal- and diagonal-axis subjects. Sixteen 2-month-olds and 14 1-month-olds provided useful data. From these 30 subjects, a total of 827 scorable EOG trials were obtained, i.e., trials on which both observers agreed upon the direction of the first eye movement and which yielded a scorable EOG record. The EOG record for each trial was scored for direction, latency, and amplitude of the first EOG potential. Slopes of EOG potentials scored indicated that all eye movements here reported were saccadic (see Figure 5). To eliminate poor EOG recordings and to deal with individual variability in the strength of the EOG potential, trials involving targets at $10 \mathrm{deg}$ were scored first for each subject, with knowledge of the observers' combined judgments of first eye-movement direction. This provided, for each subject, an indication of the amplitude of his EOG potential for a 10-deg excursion, which facilitated scoring of his 20-, 30-, and 40-deg trials. Twelve percent of the 249 10-deg trials were eliminated because of disagreement on direction of first movement between the EOG record and the observers' combined judgments. ${ }^{4}$

Each subject's 20-, 30-, and 40-deg trials were scored blind with respect to stimulus condition and observers' judgments, except that the scorer had available the amplitude of each subject's EOG potentials for all $10-\mathrm{deg}$ trials. Approximately $21 \%$ of the $57820-, 30-$, and 40-deg trials were eliminated because of disagreement between EOG scoring and the observers' judgments of direction of first movement. This error rate did not significantly vary by stimulus condition beyond $10 \mathrm{deg}$ but was slightly higher than on 10-deg trials, since the EOG scorer could not appeal to observers' judgments in cases where the EOG record was ambiguous.
Figures 3 and 4 illustrate percentage histograms of latencies, in $500-\mathrm{msec}$ intervals, to initiate a directionally appropriate first saccade across the 10-sec trial duration. On replacement trials (Figure 3), an appropriate first eye movement was generally initiated within 2 sec following offset of the central fixation stimulus at all target distances. ${ }^{5}$ One-month-olds executed first saccades with median latencies of $800,1,320$, and $1,480 \mathrm{msec}$ to targets at 10,20 , and $30 \mathrm{deg}$, respectively. Medians tests revealed that latencies on $10-\mathrm{deg}$ trials were significantly shorter than on 20-deg trials $\left(\chi^{2}=10.5\right.$ $\mathrm{df}=1, \mathrm{p}<.01)$, or on 30-deg trials $\left(\chi^{2}=16.6\right.$, $\mathrm{df}=$ $1, \mathrm{p}<.001)$, or on control trials $\left(\chi^{2}=18.9, \mathrm{df}=1\right.$, $\mathrm{p}<.001)$. There were no significant latency differences between 20-deg, 30-deg, or control trials.

Two-month-olds executed first saccades with median latencies of 480,920 , and $1,280 \mathrm{msec}$ on 10 -, 20-, and 30-deg trials, respectively. Medians tests revealed that latencies on 10-deg trials were significantly shorter than on 20-deg trials $\left(\chi^{2}=3.85\right.$, $\mathrm{df}=1, \mathrm{p}<.05)$, or on 30-deg trials $\left(\chi^{2}=28.9\right.$, $\mathrm{df}=$ $1, \mathrm{p}<.001)$, or on control trials $\left(\chi^{2}=17.0, \mathrm{df}=1\right.$, $\mathrm{p}<.001)$. Latencies on 20-deg trials were shorter than on control trials $\left(\chi^{2}=9.13\right.$, df $\left.=1, \mathrm{p}<.01\right)$, but $30-\mathrm{deg}$ and control trial latencies did not differ significantly. Two-month-olds exhibited significantly shorter latencies than 1-month-olds on 10-deg trials $\left(\chi^{2}=5.3, \mathrm{df}=1, \mathrm{p}<.05\right)$ and on 20-deg trials $\left(\chi^{2}=\right.$ 4.27. df $=1, p<.05)$, but no age difference was found on 30-deg trials.

Inspection of latencies on 10-deg trials in the replacement condition suggested the occurrence of a considerable number of saccades with latencies less than $500 \mathrm{msec}$. The frequency of initiating a saccade within $500 \mathrm{msec}$ following the onset of a 10-deg trial was significantly higher than on control trials at both $1\left(\chi^{2}=4.57, \mathrm{df}=1, \mathrm{p}<.05\right)$ and $2\left(\chi^{2}=29.44\right.$, df $=1, \mathrm{p}<.001)$ months of age. A similar effect was

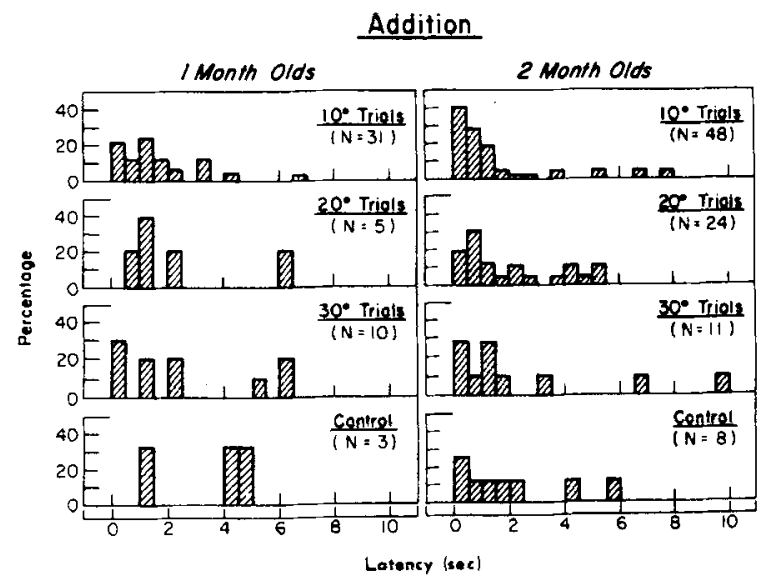

Figure 4. Frequency histogram of EOG latencies, in 500-msec intervals, to initiate a directionally appropriate first eye movement on addition trials for 1 - and 2 -month-olds. $\mathbf{N}=$ number of trials. 
true for 2 -month-olds $\left(\chi^{2}=4.89\right.$, df $\left.=1, p<.05\right)$, but not for 1-month-olds, on 20-deg trials.

On addition trials, the subjects initiated directionally appropriate first saccades only infrequently on all but 10-deg trials (Figure 4). As on replacement trials, an appropriate first saccade was generally initiated within $2 \mathrm{sec}$ following the onset of a 10-deg trial, and very often within $500 \mathrm{msec}$, especially for 2-month-olds. A medians test revealed that 2-month-olds showed shorter latencies than $1 \cdot$ month-olds on $10-$ deg trials $\left(\chi^{2}=4.19\right.$, df $=1$, $\mathrm{p}<.05)$. Directionally appropriate first eye movements on 20-deg, 30-deg, and control trials for the addition condition were too infrequent to allow us to draw any firm conclusions regarding latency distributions.

\section{The Form of Localizing Saccades}

Infants showed considerable variation in the form

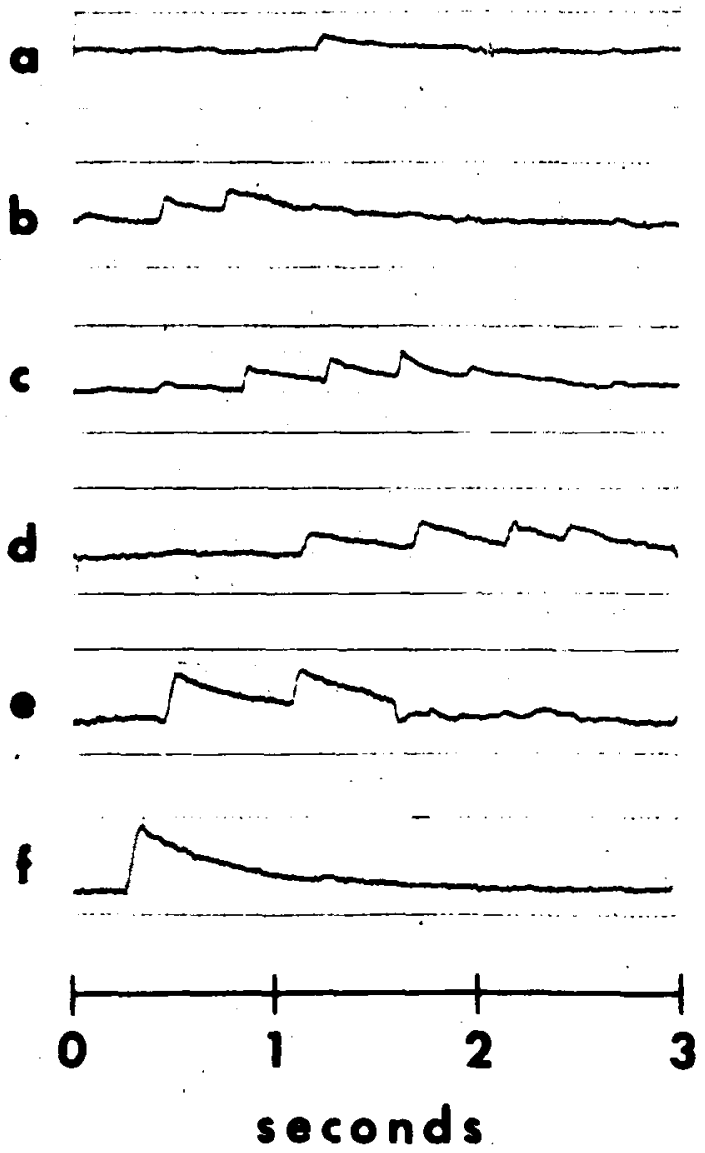

Figure 5. Examples of infant EOG records: (a) a single saccade to a target at $10 \mathrm{deg}$, (b) a double saccade to a target at $20 \mathrm{deg}$, (c) a triple saccade to a target at $30 \mathrm{deg}$, (d) a quadruple saccade to a target at $40 \mathrm{deg}$, (e) a double saccade to a target at $30 \mathrm{deg}$ followed by a return movement; and ( $f$ ) an adult record of a single saccade to a target at $30 \mathrm{deg}$. Time marks represent seconds after target onset. of directionally appropriate saccades initiated toward peripheral targets. These saccades could be (a) single, presumably normometric, ${ }^{6}$ with no detectable corrective movement (Figure 5a), (b) hypometric, with one or more subsequent saccades in the same direction (Figures $5 b, 5 c$, and $5 d$ ), or (c) hypermetric, followed by a return movement (Figure 5e). Single and hypometric saccades were by far the most typical movements; hypermetric saccades occurred on only $19 \%$ and $14 \%$ of scorable EOG trials for the $1-$ and 2-month-olds, respectively. ${ }^{7}$

Relative Frequency of Single vs. Multiple Saccades. A single localizing saccade was detined as a directionally appropriate first saccade with no detectable subsequent movements occurring within $2 \mathrm{sec}$ following saccade initiation. A hypometric saccade was defined as a directionally appropriate first saccade followed by one or more additional saccades in the target direction, each occurring within $2 \mathrm{sec}$ of the previous movement. It seemed reasonable to assume that saccades spaced within 2 sec of one another were probably part of the same attempt at localization. As we shall see, data related to the summed polarity of eye-movement potentials in a localizing series made this a tenable assumption.

It appeared that the subjects would often localize the target by means of a series of hypometric saccades, all directionally appropriate. For example, Figure $5 \mathrm{~b}$ shows a double saccade to a target $20 \mathrm{deg}$ from field center, Figure $5 c$ a triple saccade on a 30-deg trial, and Figure 5d a quadruple saccade on a 40-deg trial. In order to compare the relative incidence of single vs. multiple saccadic localizations, the number of each type of localizing saccade (single, double, triple, etc.) for each subject was expressed as a proportion of the total number of trials on which he made a directionally appropriate first saccade at each target distance. Proportions thus obtained for each type of localizing saccade were averaged to provide a group estimate of relative incidence. It may be seen in Figure 6 that for targets at 10 deg most localizations

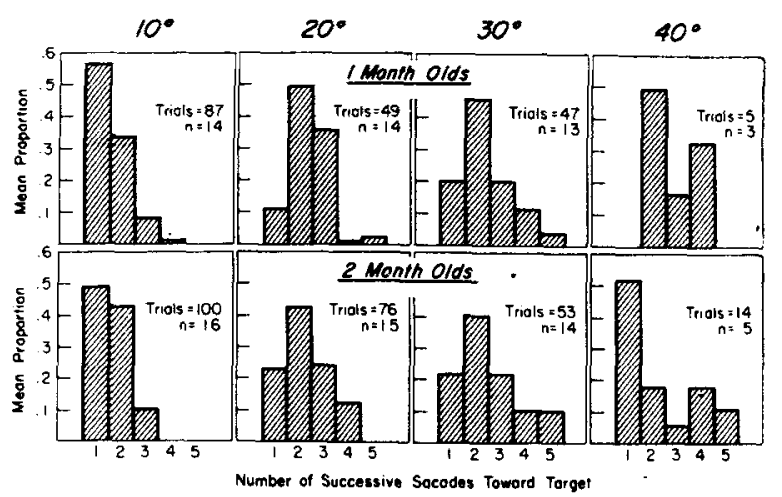

Figure 6. Mean proportions of single and multiple saccades as a function of target distance for 1- and 2-month-olds. 


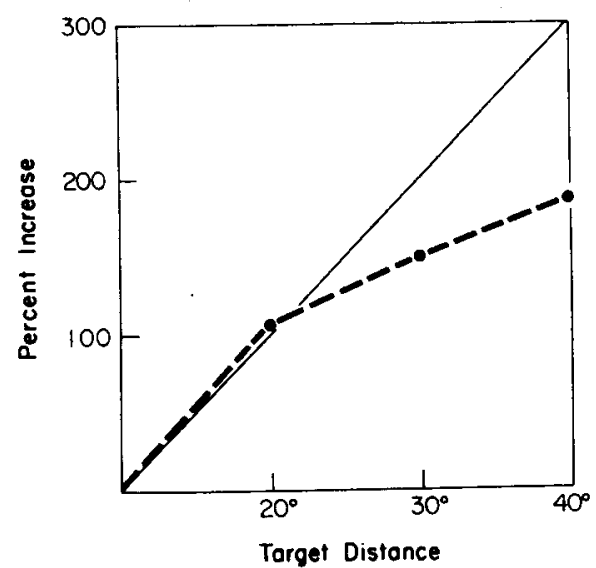

Figure 7. Mean EOG amplitude of all single and multiple saccades per trial (total eye rotation) for each target distance expressed as the percent increase over the mean EOG amplitude on 10-deg trials.

consisted of a single or double saccade. Beyond $10 \mathrm{deg}$, it appeared that ${ }^{\circ}$ the relative incidence of single saccades decreased and multiple saccades increased, although frequency distributions for targets at $\mathbf{4 0} \mathrm{deg}$ may have been unstable because of the limited number of observations. Finally, no obvious differences were apparent between 1- and 2-month-olds.

As indicated in Figure 6, for 1-month-olds $57 \%$ of 10-deg localizations were single saccades while $43 \%$ were multiple saccades. For targets at 20, 30, and $40 \mathrm{deg}$, single saccades occurred on only a mean of $14 \%$ of all trials, while multiple saccades occurred on $86 \%$ of all trials. Similarly, for 2 -month-olds, $49 \%$ of 10-deg localizations were single saccades and $51 \%$ were multiple saccades, while for targets at 20,30, and $40 \mathrm{deg}$ combined, a mean of $24 \%$ were single saccades and $76 \%$ were multiple saccades. For a significant number of subjects, the proportion of single vs. multiple saccades decreased on target trials at $20 \mathrm{deg}$ and beyond as compared with 10-deg trials (sign test: $\mathrm{p}<.001$ for 1 -month-olds; $\mathrm{p}=.006$ for 2-month-olds). Thus, all subjects showed evidence of multiple saccades at each target distance, and the likelihood of single saccades diminished with increasing target distance from the central fixation stimulus.

Amplitudes of Successive Saccades. It appeared from infant EOG recordings (Figure 5) that the amplitude of each successive potential in a multiple saccade to target was approximately equal..$^{8} \mathrm{~A}$ variety of post hoc analyses were conducted to allow some conclusions to be drawn as to whether this was the case. First, if this were true, one would expect a greater number of successive saccades in a given multiple saccade series as peripheral target distance from the central fixation stimulus increased. As indicated in Figure 6, when target distance increased from 10 to $20 \mathrm{deg}$ and beyond, the mean proportion 'of multiple saccades also increased.
However, there did not appear to be any differences in the relative frequency of multiple saccade types on 20-, 30-, and 40-deg trials.

Second, an attempt was made to determine the extent to which linearity in the EOG potential was present for rotation of the infant's eye. We have been assuming that infants, on the average (a) fixated the central fixation stimulus when each trial began, and (b) reached the peripheral target following the execution of a single, or a series of, directionally appropriate saccades. To verify these assumptions, the sum of each subject's EOG potentials in the single or multiple saccade series used to localize the target were obtained for each trial. These sums were averaged across trials by distance for each subject to provide his average total EOG potential change for targets at different distances. For each subject, the average change in potential to a target at 20,30 , or $40 \mathrm{deg}$ was then expressed as a percent increase over his average change in potential to a target at $10 \mathrm{deg}$. This was done to remove the variability between subjects in the absolute level of EOG potentials. These percentages were then averaged across subjects to provide an indication of the extent to which EOG amplitude was linearly related to eye rotation for infants in the study. Figure 7 shows that the linearity in mean EOG amplitude was the case out to, but not beyond, a $20-\mathrm{deg}$ target distance.

A third analysis was conducted to examine whether the amplitudes of successive saccades in a given localizing series were approximately equal. Since there appeared to be a linear relationship between the EOG potential and amount of eye rotation out to $20 \mathrm{deg}$ from field center, the mean amplitude of each subject's first, second, third, and fourth successive movements in a given multiple saccade were averaged for only $10-$ and 20 -deg trials. Prior to averaging, the amplitude of each saccade in a localizing series was expressed as a percentage of the first saccade in the series. Figure 8 indicates that successive movements

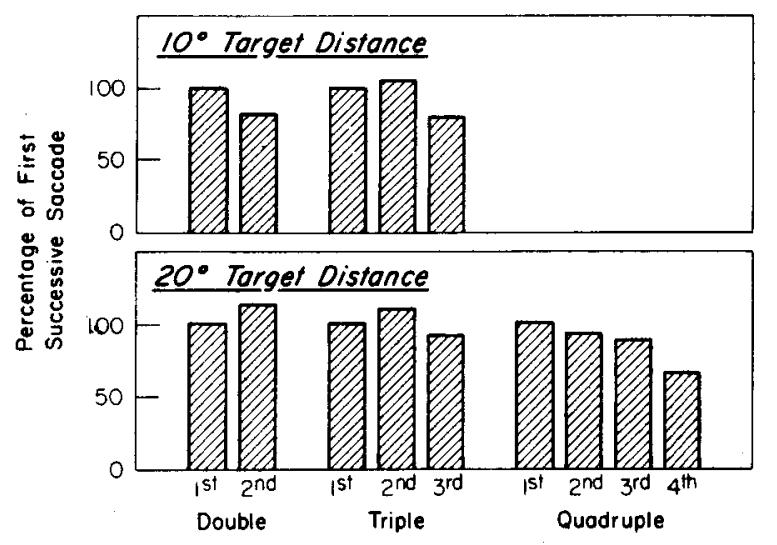

Figure 8. Mean amplitude of second, third, and fourth saccade of a multiple saccade series expressed as a percentage of the first saccade of the series. 
were approximately equal in amplitude, with only a slight trend toward decreasing amplitude as the line of sight approached the target. 9

One might assume from Figure 8 that infants typically moved their eyes a relatively fixed angular distance on each saccade of a multiple saccade series to any target presented within $20 \mathrm{deg}$ of field center. To further evaluate this possibility, we obtained an estimate of the total amount of eye rotation for directionally appropriate saccades to targets at 10 and $20 \mathrm{deg}$. Each subject's average total EOG potential from initiation to termination of localization was obtained separately for single, double, triple, and quadruple saccades to each target distance. The within-subject averages thus obtained were then averaged across subjects to provide a between-subject average of total change in EOG potential (total eye rotation) for single and multiple saccades to each target. In Table 1, the average total EOG potential change that occurred for each type of localizing saccade series to each target distance is expressed as a multiple of the average total EOG potential change that occurred when the subjects localized a target $10 \mathrm{deg}$ from field center by means of a single saccade. For simplicity, the 10-deg single saccade value was set at unity.

It may be seen from Table 1 that the average total EOG potential to a target at a given distance roughly doubled if a double rather than a single saccade was executed. The results for triple saccades are not an exact triplicate of the single saccade at that distance, but are generally in that direction, i.e., at the very least, values for triple saccades are considerably greater than for double saccades. These results are generally in accord with the notion that the infants generally executed saccades of a fixed amplitude, since it appears from the within-subject summed EOG potential that when double or triple saccades were executed, the eyes rotated considerably farther than when single saccades were executed, at a given distance. Why this should be the case with a seemingly constant initial and terminal position in the field will be later discussed.

However, certain of the findings presented in Table 1 are not consistent with the hypothesis of a fixed amplitude saccadic programming system in the young infant. Specifically, it should be noted that when average summed EOG potentials are compared across rather than within target distances, it is inescapably the case that the infant appropriately increases the amplitude of his single saccadic unit. Thus, a single eye movement to a target at $20 \mathrm{deg}$ was on the average, assuming EOG linearity, twice as large as a single saccade to a target at $10 \mathrm{deg}$. Similarly, since the average summed potentials for multiple saccades to targets at 20 deg were larger than the summed potentials for multiple saccades to $10 \mathrm{deg}$, it follows that each of the components within
Table 1 Mean Relative Amplitude of Summed EOG Potentials

\begin{tabular}{lcccr}
\hline $\begin{array}{l}\text { Type of } \\
\text { Saccade }\end{array}$ & $\begin{array}{c}\text { 10-Deg } \\
\text { Distance }\end{array}$ & $\mathrm{N}$ & $\begin{array}{c}\text { 20-Deg } \\
\text { Distance }\end{array}$ & $\mathrm{N}$ \\
\hline Single & 1.00 & 26 & 2.06 & 16 \\
Double & 1.99 & 26 & 2.97 & 25 \\
Triple & 2.76 & 11 & 3.55 & 21 \\
Quadruple & & & 4.81 & 9 \\
\hline
\end{tabular}

Note-Means indicate the amplitude of the total EOG excursion (eye rotation) per trial relative to the single saccade type on 10-deg trials. $N=$ number of infants.

multiple saccades to targets at 20 deg were larger than components of multiple saccades to targets at 10 deg. ${ }^{10}$ In sum, it appears that whereas only saccades of approximately similar average amplitude were executed in a single or multiple series at a given target distance, the size of the saccade unit in both single and multiple saccades increased as target distance increased.

Intersaccade Intervals. Mean intersaccade intervals were calculated between movements in double, triple, and quadruple saccades across all target distances. The mean latency to initiate the first movement of a multiple saccade series was also calculated. However, all latencies to first movement greater than $2 \mathrm{sec}$ were eliminated so that comparisons with later intersaccade intervals would not be biased, since by definition, intersaccade intervals in multiple saccade localizations could not exceed 2 sec. Table 2 presents the mean latencies and intersaccade intervals for double, triple, and quadruple saccades. For all types, the mean latencies to initiate a first saccade were longer than later intersaccade intervals for both 1- and 2-month-olds. Mean intersaccade intervals did not differ by position within a multiple saccade series. Thus, it appeared that once the first directionally appropriate movement was initiated, the latency to initiate further movements in the same direction decreased to a uniform level. A similar effect is noted in adults (Becker, 1972; Becker \& Fuchs, 1969; Weber \& Daroff, 1971). One-month-olds exhibited consistently longer intersaccade intervals than 2-month-olds $(t=2.54, \mathrm{df}=57, \mathrm{p}<.01)$.

\section{DISCUSSION}

The results of this study indicate that young infants are capable of rapidly initiating a saccadic eye movement toward a single extrafoveal target. The first eye movement after peripheral target onset is reliably directionally appropriate to targets as far as $30 \mathrm{deg}$ from the line of sight, but typically grossly hypometric to targets further than $10 \mathrm{deg}$ from the line of sight. Even to a target only $10 \mathrm{deg}$ distant, at least $50 \%$ of first localizing saccades are hypometric. Contrary to an earlier report (Tronick, 1972) based on a paradigm in which a central stimulus remained on during 
Table 2

Mean Latencies to First Eye Movement and Mean Intersaccade Intervals

\begin{tabular}{|c|c|c|c|c|c|c|c|}
\hline \multirow[b]{2}{*}{$\begin{array}{l}\text { Type of } \\
\text { Multiple } \\
\text { Saccade }\end{array}$} & \multirow[b]{2}{*}{$\begin{array}{l}\text { Position of } \\
\text { Saccade } \\
\text { in Series }\end{array}$} & \multicolumn{3}{|c|}{1 Month Olds } & \multicolumn{3}{|c|}{2 Month Olds } \\
\hline & & $\begin{array}{l}\text { Latency or Inter- } \\
\text { saccade Interval } \\
\text { (msec) }\end{array}$ & $\mathbf{N}$ & $\begin{array}{c}\text { Number } \\
\text { of } \\
\text { Trials }\end{array}$ & $\begin{array}{l}\text { Latency or Inter- } \\
\text { saccade Interval } \\
\text { (msec) }\end{array}$ & $\mathbf{N}$ & $\begin{array}{c}\text { Number } \\
\text { of } \\
\text { Trials }\end{array}$ \\
\hline Double & 1 st & 1059 & 13 & 60 & 756 & 15 & 74 \\
\hline Double & 2nd & $526 * * * *$ & 13 & 60 & $441 * * * *$ & 15 & 74 \\
\hline Triple & 1 st & 934 & 9 & 26 & 886 & 12 & 35 \\
\hline Triple & 2nd & $520 * *$ & 9 & 26 & $453^{* * * *}$ & 12 & 35 \\
\hline Triple & $3 r d$ & $514^{* * * *}$ & 9 & 26 & $432 * * * *$ & 12 & 35 \\
\hline Quadruple & $1 \mathrm{st}$ & 1133 & 6 & 7 & 923 & 8 & 13 \\
\hline Quadruple & 2nd & $703^{*}$ & 6 & 7 & $452 * *$ & 8 & 13 \\
\hline Quadruple & $3 r d$ & $477 * * * *$ & 6 & 7 & $380 * * *$ & 8 & 13 \\
\hline Quadruple & 4 th & $527 * * *$ & 6 & 7 & $384 * * *$ & 8 & 13 \\
\hline
\end{tabular}

* $t$ test indicated that the intersaccade interval was shorter than the initial latency to first saccade, $p<.05$.

${ }^{* *} p<.025 \quad * * * p<.01 \quad * * * p<.005 \quad N=$ number of infants.

peripheral target presentation, our results indicate that infants can initiate localizing eye movements very rapidly if the central stimulus disappears at the time of peripheral target onset. This latter result corroborates similar findings recently reported by Harris and MacFarlane (1974).

Tronick's data also overestimated the reaction time of the infant saccadic system by including observer reaction time in the mean latency to target localization. He also included trials on which the infant did not direct his first eye movement toward the target, but subsequently localized it. We felt (a) that only trials on which the first eye movement was directed towards the target were strong evidence that the target had been detected prior to movement, and (b) that the latency of the first movement was the best estimate of quickest saccadic latency. The resulting latency distributions we obtained were much more variable than typical adult examples in the literature (Komoda et al., 1973; Saslow, 1967; Wheeless et al., 1966), but much of this variability undoubtedly stemmed from our inability to "instruct" the infant as we would an adult. The EOG latency data indicated that despite the infant's higher variability in the time taken to initiate a directionally appropriate saccade, a significant proportion of infant latencies fell within a range of 240 to $500 \mathrm{msec}$.

Infants exhibited a very large number of directionally appropriate first saccades. However, at least half of these, unlike the adult, were grossly hypometric, and followed by one or more large additional saccades toward target. Could these multiple saccades have been artifacts? First, it could be argued that multiple saccades were an artifact of head restraint. This argument is unlikely because (a) head restraint was minimal, with several infants occasionally exhibiting both head and eye movements, (b) head movements could be detected on the EOG record as a slower potential change usually present as a compensatory movement in the opposite direction to the initial saccade, (c) multiple saccades were evident at all target distances, including 10-deg trials where saccadic eye movements do not typically involve head movements in adults, and (d) adults do not show such grossly hypometric saccades even with severe head restraint. One might argue, however, that infants attempted to program a combination of eye and head movement, but because of immature head control only the eye rotated, causing the undershoots obtained. In this sense, head movement could have affected the results, but in a nonartifactual sense.

Second, it could be argued that the infants were not strongly "motivated" to localize the peripheral targets, and lazily jerked their eyes toward target. This argument appears unlikely since first eye movements on over $90 \%$ of all $10-\mathrm{deg}$ replacement trials were directionally appropriate (see Figure 3 ), indicative of high interest in the display, especially since infants, unlike tired adults, had not been instructed to localize the target. Moreover, at a 10-deg target distance, multiple saccades appeared on approximately $50 \%$ of all trials, similar to the incidence on trials at further distances where appropriate localizing response rates were lower. In addition, the linearity of the EOG potential for targets at 10 and $20 \mathrm{deg}$ provides support that these targets were actually looked at (assuming a linear relation between size of EOG potential and amount of eye rotation for $\pm 20 \mathrm{deg}$ ). For the foregoing reasons, it seems most unlikely that the infants were not motivated to localize the targets. This, of course, does not rule out the possibility that the attentional system of the infant was dissimilar from that of the adult because of immaturity, e.g., infants in this study may have been localizing on a more reflex basis.

The data presented in Figure 8 suggested that, for infants, the amplitude of successive saccades within a multiple saccade series was approximately equal. If this were true, one would expect the same number of saccades within a multiple saccade series to be used in 
localizing targets at the same distance from the line of sight. However, the large size (approximately $4 \mathrm{deg}$ ) of the targets used in the present study (chosen so as to maximize the probability of movement) may have resulted in variation across trials in the number of saccades used to localize a target at the same distance (see Figure 6). For a target at $10 \mathrm{deg}$, whether a single or whether a double saccade occurred may have been the result of variation in starting and terminal eye position. For example. if the line of sight of an infant was originally at the right- or leftmost contour of the central fixation stimulus and moved either to the leftor rightmost contour (respectively) of a peripheral target $10 \mathrm{deg}$ away, the angular distance of eye excursion would have been either 6 or $14 \mathrm{deg}$, respectively. Thus, variation in the number of saccades used to localize targets at the same distance may have been at least partially the result of variation in starting and terminal position, a single saccade being executed for shorter starting-terminal distances.

However. it was also found that an infant could increase the size of the unit saccade across target distances. even though the size of the unit remained relatively fixed within a multiple saccade series on a given trial. For example, a given infant could execute two 5-deg saccades to reach a target $10 \mathrm{deg}$ away and also two 10-deg saccades to localize a target $20 \mathrm{deg}$ aw ay. Apparently, a retinal estimate of target distance results in the programming of a distance-related unit of saccade amplitude that is reapplied until the target is localized. Although we were unable to exactly calibrate the infant EOG signal so that it could be converted to visual degrees of eye rotation, our best guess as to the size of the unit saccade of a multiple saccade series would be 6 to $7 \mathrm{deg}$ to targets $10 \mathrm{deg}$ distant and up to $15 \mathrm{deg}$ to targets beyond $10 \mathrm{deg}$.

It is clear, then, that the infant generally programs and executes a distance-related first saccade to a peripheral target. However, unlike the adult (Becker \& Fuchs, 1969; Weber \& Daroff, 1971), approximately $50 \%$ rather than $90 \%$ of the angular distance to target is traversed by the infant's first eye movement. Still more puzzling is the finding that the second eye movement of a localizing series is approximately equal in magnitude to the first. It would appear that the second eye movement is programmed and executed on the basis of the distance between line of sight and target prior to, rather than following, the first movement. The infant appears to reapply a distance-related fixed unit of eye excursion to reach target. However, the fact that normometric first saccades to 10-deg targets occurred with considerable frequency suggests that once the line of sight is within approximately $10 \mathrm{deg}$ or less of target, a more distance-specific terminal movement may be programmed to reach target. Before the foregoing description can be more specifically stated, however, exact data regarding peripheral localization, where actual starting and terminal positions from the line of sight are objectively specified, are needed.

\section{REFERENCES}

Al.PERN. M. Effector mechanisms in vision. In J. W. Kling and L. A. Riggs (Eds.). Woodworth and Schlosberg's Experimental psychology (3rd ed.). New York: Holt, Rinehart. \& Winston, 197i. pp. 369-394.

BaCh-Y-Rita. P.. \& Collins, C. C. The control of eye movements. New York: Academic Press. 1971.

BAkTz. A. E. Eye movement latency, duration, and response time as a function of angular displacement. Journal of Experimental Psychólogy, 1\%2. 64, 318-324.

BARtz. A. E. Fixation errors in eye movements to peripheral stimuli. Joumal of Experimental Psychology, 1967. 75. 444-446.

BeCKER. W. The control of eye movements in the saccadic system. In J. Dichgans and E. Bizzi (Eds.), Cerebral control of tye movements and motion perception. A symposium held in conjunction with the 25th International Congress on Physiolngical Science. Munich, 1971. Bibliotheca Ophthalmologica. 1972, 82. 233-243.

Becker, W.. \& Fuchs. A. F. Further properties of the human saccadic system: Eye movements and correction saccades with and without visual fixation points. Vision Research. 1969. 9. 1247-1258.

Bond. E. K. Perception of form by the human infant. Psychological Bulletin. 1972. 77. 225-245.

Davson, H. The physiology of the eye. New York: Academic Press. 1972.

DArton, G. O.. \& Jones, M. H. Analysis of characteristics of tixation reflex in infants by use of direct current electrooculography. Neurology. 1964, 14. 1152-1156.

Dichoans, J., \& Brzzi, E. (Eds.) Cerebral control of eye movements and motion perception. Symposium held in conjunc. tion with the 25th International Congress on Physiological Sciences, Munich, 1971. Bibliotheca Ophthalmologica, 1972. 82. 1.403

Grunin, R., \& Mostofsky, D. I. Eye movement: A bibliographic survey. Perceptual and Motor Skills, 1968, 26, 623-639.

Harris, P.. \& MacFariane, A. The growth of the effective visual field from birth to seven woeks. Journal of Experimentul Child Psychology, 1974, 18. 340-348.

Kessen. W.. Haith. M., \& Salapatek, P. Infancy. In P. H. Mussen (Ed.). Curmichael's manual of child psychology (3rd ed.). New York: Wiley, 1970. Pp. 287-446.

Kessen. W., Salapatek. P., \& Haith, M. The visual response of the human newborn to linear contour. Joumal of Experimental Child Psychologi, 1972, 13, 9-20.

Komoda, M. K.. Festinger, L., Phillips, L. J., Duckman, R. H., \& Young, R. A. Some observations concerning saccadic eye movements. Vision Research, 1973, 13, $1009-1020$.

Leushina. L. I. On estimation of position of photostimulus and eye movements. Biofizika, 1965, 10. 120-136.

Mann. I. The development of the human eye. New York: Grune and Stratton, 1964.

Mclaughlin. S. C. Parametric adjustment in saccadic eye movements. Perception \& Psychophysics, 197, 2, 359-362.

RAshrass, C. The relationship between saccadic and smooth tracking eye movements. Journul of Physiology, London, 1961. 159. 320-338.

Salapatek, P. Visual scanning of geometric figures by the human newbom. Joumal of Comparative and Physiological Psychology. 1968. 66. 247-258.

Salapatex, P., \& Kessen, W. Visual scanning of triangles by the human newborn. Journal of Experimental Child Pspichology, 1906, 3, 155-167. 
SAsLow. M. Latency for saccadic eye movements. Journal of the Optical Society of America, 1\%7, 57, 1030-1033.

Stechler, G., \& Latz, E. Some observations on attention and arousal in the human infant. Journal of the American Acade'my of Child Psychiatrists, 1966, 5, 517-525.

Todd, T. W., Beecher, H., Williams, G. H., \& Todd, A. W. The weight and growth of the human eyeball. Human Biology, 1940, 12, 1-20.

Tronick. E. Stinulus control and the growth of the infant's effective visual tield. Perception \& Psychophysics, 1972, 11. 373-376.

Tronick, E., \& Clanton, E. Infant looking patterns. Vision Reseurch, 1971, 11, 1479-1486.

Weber, R. B., \& Daroff, R. B. The metrics of normal human saccadic eye movements. Vision Reseanch, 1971, 11, 921-928.

Westheimer. G. Mehanism of saccadic eye movements. Archives of Ophthalmology, 1954, 52, 710-724.

Wheeless, L., Boynton, R., \& Cohen, G. Eye-movement responses to step and pulse-step stimuli. Journal of the Optical Society of America, 1966, 56, 956-960.

White, C. T., EAson, R. G., \& Bartlett, N. R. Latency and duration of eye movements in the horizontal plane. Journal of the Optical Society of America. 1962, 52, 210-213.

Wilmer, H. A., \& SCammon, R. E. Growth of the components of the human eyeball. Archives of Ophthalmology, 1950, 43, 599-619.

\section{REFERENCE NOTES}

1. Ames. E. W., \& Silten, C. K. Methodological issues in the study of age differences in infant's attention to stimuli varying in movement and complexity. Paper presented at the biennial meeting of the Society for Research in Child Development. Minneapolis. Minnesota, 1965.

2. Mundy-Castle, A., \& Anglin, J. M. Looking strategies in infants. Paper presented at the biennial meeting of the Society for Research in Child Development. Santa Barbara, California, 1969.

3. Salapatek. P. The visual investigation of geometric pattern by the one and two month old intant. Paper presented at meetings of the American Association for the Advancement of Science, Boston. December 1969

\section{NOTES}

1. Dayton and Jones (1964) recorded refixation saccades to a moving stimulus of up to $286 \mathrm{deg} / \mathrm{sec}$ at 1 month of age.

2. There were significantly different baseline probabilities of movement on center-on vs. center-off control trials for each axis group (Tukey $99 \%$ confidence intervals). In other words, during center-on control trials, the subjects typically continued to look at the central fixation stimulus $(72 \%$ of all trials), while during center-off control trials the subjects tended to search the stimulus tield, only rarely continuing to look at the empty center of the field ( $8 \%$ of all trials).

3. In absolute terms, probabilities of a directionally appropriate saccade were consistently much higher on replacement than on addition trials. However, the high probability of eye movement to either semifield during center-off control trials masked this effect. A more stringent response criterion of "directionally appropriate saccade" on all trials, e.g., one in which a location criterion for terminal line of sight was required, undoubtedly would result in a lower center-off baseline and a larger additionreplacement effect.

4. Signal/noise ratio of the EOG signal varied considerably among subjects. Therefore, only trials on which all three indicators (EOG and both observers) agreed on the direction of first eye movement were used.

5. The results from $40-\mathrm{deg}$ trials are not shown since a directionally appropriate scorable saccade occurred on only 17 such trials.

6 . Evidence later presented will support the contention that single saccades were typically normometric to targets within $20 \mathrm{deg}$ of field center.

7. Adults who were placed in the apparatus and instructed to "look at the light that comes on" exhibited only single saccades to target on all 192 trials with no observable corrective movements (see Figure 5f). Of course, it should be recalled that our EOG recording was sensitive only to movements of approximately $2 \mathrm{deg}$ or more, and that peripheral targets were much larger than those used in most adult studies.

8. For adults instructed to localize targets out to $20 \mathrm{deg}$, such a pattern of equal EOG potentials would indicate a sequence of eye movements of equal rotation. Linearity typically breaks down past $20 \mathrm{deg}$, but it is easily possible to calibrate the EOG signal for more distance points. However, it was not possible to calibrate the EOG signal of each infant since he could not be instructed to direct his line of sight reliably to known points in the field. In fact. investigation of his ability to do so was the purpose of this experiment. Moreover. there was little reason to expect a priori that the infant's EOG signal was linearly related to eye rotation because of large structural differences between the adult and infant eye. In short, it was unknown whether, or across what excursion, there existed for the infant linearity between the EOG signal and amount of eye rotation.

9. For double saccades on 10-deg trials, first and second saccade amplitudes differed by only $20 \%$ or less on 55 of 78 trials. For double saccades on 20-deg trials, first and second saccade amplitudes differed by only $20 \%$ or less on 33 of 53 trials. For triple and quadruple saccades on 10- and 20-deg trials. no two saccade amplitudes in a given series differed by more than $20 \%$ on 40 of 66 trials. Thus it appeared that the amplitudes of successive saccades in a given multiple saccade series were approximately and consistently equal for targets within $20 \mathrm{deg}$ of field center.

10. It should be recalled that components in a given multiple saccade series were earlier shown to be approximately equal in amplitude (see Figure 8).

(Received for publication May 1, 1974; accepted September 30, 1974.) 